\title{
Trends in Compressive Sensing for EEG Signal Processing Applications
}

\author{
Dharmendra Gurve $^{1, *(\mathbb{C})}$, Denis Delisle-Rodriguez ${ }^{2} \mathbb{D}$, Teodiano Bastos-Filho ${ }^{2} \mathbb{C}$ \\ and Sridhar Krishnan ${ }^{1}$ (D) \\ 1 Department of Electrical, Computer, and Biomedical Engineering, Ryerson University, \\ Toronto, ON M5B 2K3, Canada; krishnan@ryerson.ca \\ 2 Postgraduate Program in Electrical Engineering, Federal University of Espirito Santo, \\ Vitoria 29075-910, Brazil; delisle05@gmail.com (D.D.-R.); teodiano.bastos@ufes.br (T.B.-F.) \\ * Correspondence: dgurve@ieee.org
}

Received: 25 May 2020; Accepted: 23 June 2020; Published: 2 July 2020

\begin{abstract}
The tremendous progress of big data acquisition and processing in the field of neural engineering has enabled a better understanding of the patient's brain disorders with their neural rehabilitation, restoration, detection, and diagnosis. An integration of compressive sensing (CS) and neural engineering emerges as a new research area, aiming to deal with a large volume of neurological data for fast speed, long-term, and energy-saving purposes. Furthermore, electroencephalography (EEG) signals for brain-computer interfaces (BCIs) have shown to be very promising, with diverse neuroscience applications. In this review, we focused on EEG-based approaches which have benefited from CS in achieving fast and energy-saving solutions. In particular, we examine the current practices, scientific opportunities, and challenges of CS in the growing field of BCIs. We emphasized on summarizing major CS reconstruction algorithms, the sparse basis, and the measurement matrix used in CS to process the EEG signal. This literature review suggests that the selection of a suitable reconstruction algorithm, sparse basis, and measurement matrix can help to improve the performance of current CS-based EEG studies. In this paper, we also aim at providing an overview of the reconstruction free CS approach and the related literature in the field. Finally, we discuss the opportunities and challenges that arise from pushing the integration of the CS framework for $\mathrm{BCI}$ applications.
\end{abstract}

Keywords: compressive sensing; EEG; low power BCIs; neurofeedback; assistive technology; sampling; data acquisition

\section{Introduction}

As the fourth industrial revolution has accelerated, big data is growing in the field of healthcare, and the field of neural engineering is no exception. The neurological big brain data comes from a number of sources including hospitals, research labs, and consumer-directed neurotechnological devices. This brain data may be used to understand the brain structure and function for improving an individual's health. For instance, the Image and Data Archive (IDA), a neurocognitive data archiving, and sharing platform, presently contains over 130 terabytes of neurological data to study the brain structure and functions [1]. Since each wire implanted in the brain tracks the activity of multiple neurons at once, it is difficult to identify which electrical bursts belong to which cell because of the biological complexity of the brain. Neuroscientists are now studying how an entire living brain functions by understanding different neuronal dynamics. For instance, one of the initial researches towards a scalable high-bandwidth brain research and an integrated model proposed by Musk [2] can collect brain signals from 3072 electrodes simultaneously. Few initial studies on other species brains 
also confirm the immensity of cortical activity. For example, 20 min of neural activity in a mouse brain recording produces about 500 petabytes of flickering data [3]. In another brain study, the brain needed a few terabytes of images to reconstruct 1000 nerve cells (less than $1 \%$ of total cells) from the Drosophila brain, and researchers spent a decade to collect data from 60,000 neurons at a rate of 1 gigabyte per cell. According to the same study, the human brain would take an estimated 17 million years [3] to collect the data from the 86 billion neurons using the same protocol. Furthermore, neural interface technology is trending towards wireless options as it has a significant advantage that the user can wear it for the long-term. Measuring neural activity throughout the day across a wide range of activities can provide a greater possibility for medical specialists to develop an effective patient-specific preventive solution. However, the more data these wireless neurotechnological devices gather, the more challenges we face, including high bandwidth, complex hardware, and more battery power required to handle the large amounts of data transferred from sensor end to decoder end of the wireless system.

As neuroscientists push the limits to understand the immense complexity of the brain with big data, researchers are developing efficient computational methods to handle and interpret the resulting data. Various data compression methods have been used for effective storage and transmission of multichannel electroencephalography (EEG) signals including, discrete cosine transform (DCT) based, discrete wavelet transform matrix (DWT) based, and run-length encoding. These methods need two independent steps, which are signal acquisition followed by compression. In contrast, compressive sensing (CS) allows data acquisition and compression simultaneously, which provide on-chip data compression and leads to efficient hardware implementation for signal acquisition. Therefore, CS has become an increasingly utilized paradigm for efficient data acquisition. The theory of CS was first introduced in [4] by Donoho et al. as a new paradigm for efficient data acquisition. The CS-based signal acquisition of an analog signal can be implemented with a continuous sensing operator that randomly sub-samples the input data and provides compressed measurements that consist of very few linear projections of the original signal. The theory of CS works well with the signals that can be represented by a significant $k$ coefficients (i.e., $k$-sparse signals) over a $N$-dimensional basis. In other words, in contrast to the Nyquist-Shannon sampling theorem, CS relies on the sparsity data, and can be used for sampling the sparse signal below the Nyquist-Shannon limit, allowing its reconstruction from much fewer samples. In addition, CS offers synergistic time, and bandwidth reduction for wireless signal transmission with sparse sampling, and hence decreases the hardware and power requirements of the sensing devices.

The theory of CS was initially proposed for low-rate image acquisition. It was then developed for many other applications such as ultrasound imaging [5], face recognition [6,7], single- pixel camera [8], wireless sensors networks [9,10], cognitive radio networks [11,12], sound localization [13], audio processing [14,15], radar imaging [16,17], image processing [18,19], and video processing [20,21]. Similarly, CS has contributed to various neural engineering research including, neuronal network connectivity [22], magnetic resonance image (MRI) acquisition [23], MRI reconstruction [24], electroencephalogram (EEG) monitoring [25], compressive imaging [26,27], and other applications.

Furthermore, the CS framework has been also extensively used in Brain-Computer Interfaces (BCIs) in dealing with many challenges and to make current EEG-based approaches faster, and more energy-efficient. This paper provides a comprehensive review of the CS-related EEG studies by summarizing the related research papers published in the last two decades in terms of the CS reconstruction algorithms, the sparse basis, and the measurement matrix used in the BCI field. This work also highlights the current state of knowledge of the CS used in EEG studies and explains the possible challenges of the current $\mathrm{BCIs}$ are facing that lead to the development of effective future CS-based BCIs. Since a systematic review of CS for BCIs is missing in the literature, and this review will be useful for researchers and practitioners.

The rest of the paper is organized as follows. The motivation to use CS for BCI applications and an overview of currently available CS-based EEG studies are in Section 2. A detailed theoretical description of CS theory is discussed in Section 3, in which we also discuss the state-of-the-art about 
reconstruction algorithms, the sparse basis, and the measurement matrix being used in current BCIs. The idea of reconstruction free learning and related studies are discussed in Section 4. In Section 5, we provide the challenges and opportunities that future CS-based wireless neurotechnological devices may encounter. Finally, we conclude the review in Section 6.

\section{Need and Applications of CS for EEG}

With recently growing progress in neural engineering research, EEG based methods have been revealed to be a promising approach for diagnosis, rehabilitation, and restoration of motor functions. In wireless EEG based devices, batteries have been used as the primary source of energy. Sophisticated tasks in practical wireless BCI applications-including signal acquisition, signal processing, and wireless EEG transmission for long term-result in heavy power consumption. Furthermore, the data recorded from multi-channel neural recording implants and neurological devices result in large storage space. The CS-based EEG compression and sensing can provide saving in signal acquisition, and transmission to facilitate signal processing in the resource constraints circumstances. Roughly, the general CS-based EEG framework can be broadly divided into four main steps (shown in Figure 1), which are signal acquisition and compression at the sensor node, and signal reconstruction, feature extraction, and decoding at the receiver node.
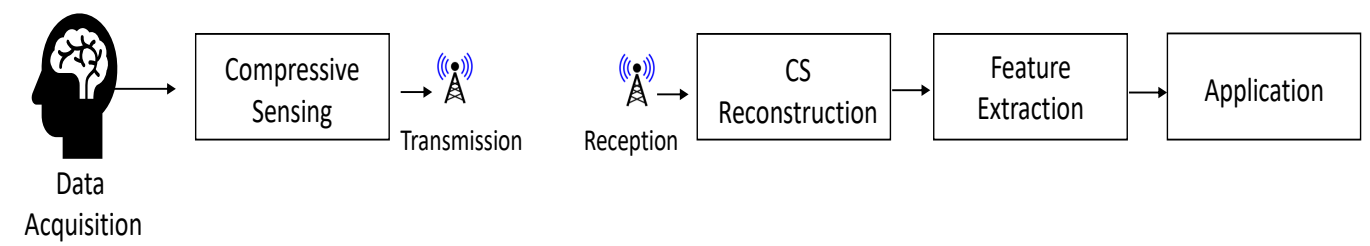

Figure 1. Block diagram of CS-based EEG studies

In this regard, the researchers around the world are working on improving these four steps and proposed various interesting applications. For instance, R. Meenu et al. [28] used the idea of CS for detecting the presence or absence of epileptic seizures in the EEG signal. The work proposed by K. Zeng et al. [29] used the compressibility calculated using the CS theory in EEG for automatic detection of seizure states. The authors used continuous wavelet transform (CWT) to extract features from time-frequency representation, related to ictal, pre-ictal, and ictal EEG. T. Moy et al. [30] proposed CS-based EEG signal acquisition and biomarker-extraction system with flexible, thin-film electronics. They successfully demonstrated the reconstruction of compressed EEG signals at up to $8 \times$ compression and spectral feature extraction for seizure detection from compressively sampled EEG signals. Another study by A. Abdulghani et al. [31] discussed the trade-offs related to the practical performance of CS for long term EEG signals. The work proposed by K. Abualsaud et al. [32] also utilized the idea of CS in wireless EEG-based epileptic seizure detection and investigated the trade-off between the complexity of CS-based framework in terms of power and classification accuracy. A few other studies [33-35] also used the theory of CS for seizure detection and showed a significant reduction in computational power and storage.

Furthermore, to address the issue of the massive amount of data originating from EEG acquisition, the study proposed by N. Mammone et al. [36] also utilized the theory of CS to compare the characteristics of the brain network organization in Alzheimer's disease (AD), mild cognitive impaired (MCI), and healthy elderly subjects by analyzing their EEG recordings. F. Morabito et al. [37] also proposed an interesting study on finding the compressibility property of EEG signals collected from AD patients, MCI patients, and healthy elderly. The finding of this study shows that it is possible to use the CS framework to compress and reconstruct the EEG signal with the signal quality required by clinical constraints, which not only reduces the throughput but also discriminate brain states among AD, MCI, and normal healthy elderly. A hardware implementation of a low power multichannel EEG signal 
acquisition system based on distributed CS is proposed by B. Kaliannan et al. [38], which enables lower power and lesser complexity in the sensing end. In order to reduce the power consumption and area overhead, M. Hosseini et al. [39] also proposed a new microelectronic system for compressive recording of multichannel high density intracranial neural signals. The performance of efficiently reconstructed multi-channel neural signals in this study is evaluated through the system and circuit-level simulations. As another application of CS in BCIs, R. Shriwastav et al. [40] proposed a novel BCI based on CS and a deep learning framework, where the authors reconstruct the motor imagery EEG signal using a convolutional neural network (CNN). The study proposed by H. Lee et al. [41] presented an automatic sleep-stage classification system by utilizing CS for EEG data compression. This work used a radial basis function (RBF) neural network for sleep-stage classification utilizing the temporal statistical and spectral features extracted from the reconstructed EEG signal. The results of this study showed that the CS-based approach achieved high classification accuracy and reduced the hardware complexity of the system with respect to the previously reported sleep-stage approaches.

\section{CS for EEG Signal}

As discussed before, CS randomly sub-samples the input data and directly generates compressed measurements while still preserve the information of interest. To understand CS mathematically, consider $\mathbf{x}$ be the signal of interest of dimension $N \times 1$. A measurement vector $\mathbf{y}$ can be computed using an $M \times N$ matrix $\boldsymbol{\Phi}$. Mathematically, the signal sampling model using the CS framework can be given by (1)

$$
\mathbf{y}=\mathbf{\Phi} \mathbf{x}
$$

where, $\mathbf{x} \in \mathbb{R}^{N}$ is the input signal, $\boldsymbol{\Phi} \in \mathbb{R}^{M \times N}$ is the CS measurement matrix and $\mathbf{y} \in \mathbb{R}^{M}$ is the compressed measurements. Furthermore, $N$ represents the number of samples in the input signal, and $M$ represents the number of measurements. In a typical CS setting $M<<N$, so that there are fewer measurements in $\mathbf{y}$ than the original signal $\mathbf{x}$.

Here, it is important to emphasize that certain conditions should be fulfilled for an efficient CS scenario, which includes input signal sparsity and incoherence between $\boldsymbol{\Phi}$ and the sparsity basis. Important methodological considerations for CS such as sparse representation, sensing matrix design, and reconstruction algorithms will be covered in detail in the following sub-sections.

\subsection{Sparse Representation}

In Equation (1), the recovery must incorporate some prior knowledge on original signal $\mathbf{x}$. The structure that is widely assumed in CS is sparsity, and, therefore, the theory of CS relies on the assumption that the signals being processed are sparse in nature. A signal $\mathbf{x}$ is called $k$-sparse if it has only a few nonzero entries (i.e., $k$ nonzero coefficients). However, for several practical applications, signals such as EEG are not explicitly sparse but near sparse. If the signals of interest are not sparse enough, it can be sparsified with respect to such overcomplete dictionaries $\boldsymbol{\Psi}$ into its transformed domain; this is known as a dictionary or sparse basis. These dictionaries obtain the sparse form of the signal by transforming it into other domains, such as wavelet, Gabor, splines, and Fourier domain. The dictionary is a fixed matrix, which maps the input signal as a sparse linear combination of its elementary signals called "atoms". These dictionaries are highly redundant, which allows us to obtain a sparser representation with a variety of atoms. A study by D. Wen et al. [42] discussed more on sparsity of an EEG signal and presented a review of sparse representation-based EEG classification methods for epilepsy detection, BCIs and cognitive impairment.

Assume that a one-dimensional discrete-time signal s of length $N$ exhibits sparsity in certain orthonormal basis $\boldsymbol{\Psi}$ defined by the basis vectors $\Psi=\left[\Psi_{1}\left|\Psi_{\mathbf{2}}\right| \Psi_{\mathbf{3}} \mid \ldots \boldsymbol{\Psi}_{\mathbf{N}}\right]$. Therefore, the signal $\mathrm{s}$ can be represented using its sparse transform domain vector $\mathbf{x}$ as follows:

$$
x=\Psi s=\sum_{i=1}^{N} s_{i} \Psi_{i}
$$


where $\mathbf{s}$ is the $N \times 1$ column vector of the signal of interest. Here, it is clear that both $\mathbf{x}$ and $\mathbf{s}$ are different representations of the same signal with time and $\Psi$ domains, respectively. The value of $\mathbf{x}$ in (1) can be replaced by $\Psi$ s using (2), and, therefore, it is obtained

$$
\begin{gathered}
\mathbf{y}=\boldsymbol{\Phi}(\Psi \mathbf{s}), \\
\mathbf{y}=\mathbf{A s},
\end{gathered}
$$

where $\mathbf{A}$ in (4) is a $M \times N$ dimension matrix, which transforms and compresses a $k$-sparse signal $\mathbf{x}$ into a $M \times 1$ measurement $\mathbf{y}$.

In the last few years, researchers have devoted lot of effort for developing a suitable dictionary that helps to find the sparser representation of the EEG signal. In the literature, the Gabor dictionary, which can be defined by Gaussian envelope sinusoidal pulses [31] is widely used as a sparse basis. For instance, the work proposed by M. Mohsina et al. [43] and S. Aviyente et al. [44] uses Gabor transform as the sparsifying basis of EEG with the focus of reducing energy consumption for processing and transmission. However, they did not consider the inter-channel correlation of the EEG signal in their study. P. Dao et al. [45] evaluated the effect of different time and frequency step sizes in building Gabor atoms [43,44] on EEG signal compression using CS. Furthermore, P. Dao et al. [46] also discussed on a quantitative comparison of CS using Gabor and K- singular value decomposition (SVD) dictionaries. Similarly, R. Kus et al. [47] also proposed a novel construction of an optimal Gabor dictionary for multichannel and multi trial EEG, which allows a priori assessment of maximum a one-step error of the matching pursuit (MP) algorithm. The work proposed by H. Zhang et al. [48] also used the over-complete Gabor dictionary for the EEG signal. Few researchers, such as L. Chen et al. [49] and R. Quian et al. [50] also used the Gabor coefficients of the EEG signals for seizure detection. Furthermore, F. Morabito et al. [37] used the classical Gabor wavelet dictionary for the dictionary functions for Alzheimer's disease (AD) analysis. They claimed that a joint use of different channels with the same approach could further improve the compressibility of EEG signals from AD patients, as AD is also known to be responsible for perturbed synchrony among channels. However, a technical issue still persisting for most of Gabor dictionary is the over complete redundant representation.

The discrete cosine transform as a dictionary basis is another commonly used approach. The study proposed by Z. Zhang et al. [51] used the idea of DCT and inverse DCT matrix as the dictionary matrix $\Psi$ to find the sparse representation of the EEG signal. In this study, the authors compared the performance of the DCT dictionary $\Psi$ in combination with a few representative CS reconstruction algorithms. Another research conducted by D. Birvinskas et al. [52] also used DCT domain representation by expressing an EEG signal as a DCT coefficient vector for signal compression. The Mexican hat function defined by the second derivative of Gaussian functions [45] as a dictionary basis is also used to find the sparse basis for the time-frequency analysis of EEG signals [53].

The research growth in the above-mentioned various sparse basis for the EEG signal offers researchers an opportunity to explore more on EEG signal sparsity. However, these methods have difficulty in reconstructing accurate EEG signals because of the apriori assumption of signal structures, and fixed basis function selection. Recently, data-driven dictionary learning has been explored to represent EEG signals into its sparse domain, which is more suitable for the sparseness of neural activity signals such as EEG. The work proposed by M. Fira et al. [54] proposed a data-driven dictionary design by building the dictionary using the EEG signal from the training dataset itself rather than using any fixed dictionary. Furthermore, apart from these mainstream dictionaries for EEG signal, few other studies also used B-Spline dictionary [55,56], linear and cubic-Spline dictionaries [57], Spline dictionary [58], Meyer wavelet function dictionary [59], or Daubechies wavelets function dictionary [60] to obtain the sparse representation of the input signal. 


\subsection{Sensing Matrix}

An efficient design of suitable $\boldsymbol{\Phi}$ is one of the important aspects of the CS framework because $\boldsymbol{\Phi}$ plays an important role for the compression of $\mathbf{x}$ at the sensor end and its reconstruction from $\mathbf{y}$ at the receiver end with given $\boldsymbol{\Phi}$ and $\boldsymbol{\Psi}$. A variety of sensing matrices such as random sensing matrices, deterministic sensing matrices, and structural sensing matrices are proposed in the literature. Figure 2 shows a broad classification of available sensing matrices. However, most of the sensing matrices are designed with the assumption that the signal $\mathbf{x}$ is explicitly sparse, which is not the case for the EEG signal.

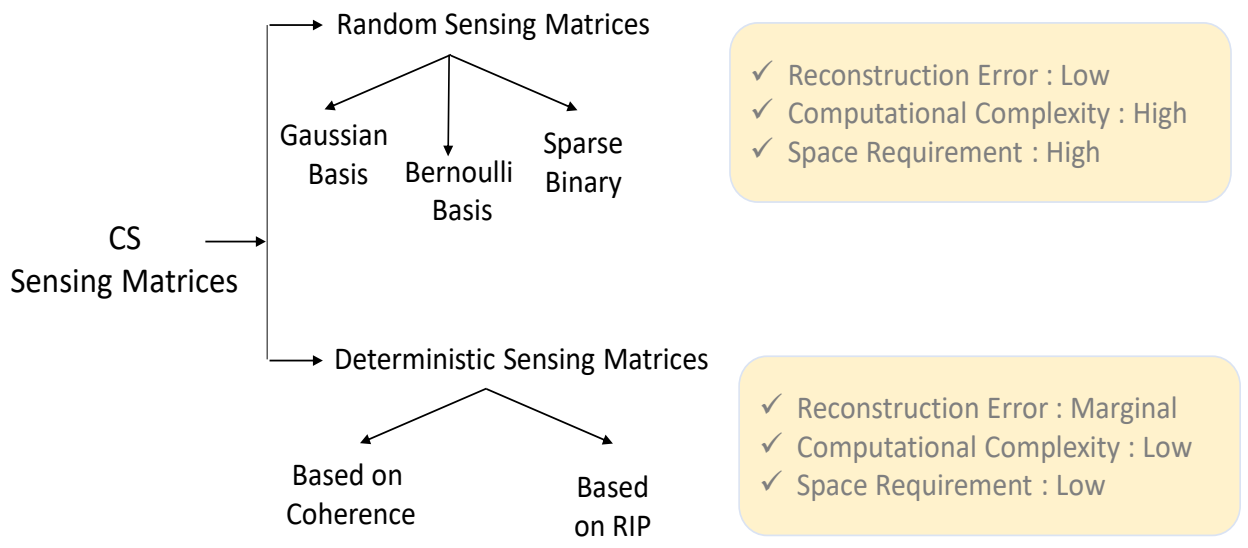

Figure 2. Classification of sensing matrices

A primary criterion for CS success is the incoherence between the sampling basis $\Psi$ and the sensing basis $\boldsymbol{\Phi}$, i.e., $\boldsymbol{\Phi}$ must be maximally incoherent with the sparsifying dictionary $\boldsymbol{\Psi}$, and, therefore, the researchers have focused on designing the sensing matrix $\boldsymbol{\Phi}$ to be a random matrix so that it becomes nearly orthogonal. To this end, various random distribution functions, such as Gaussian or Bernoulli distributions are used to design a random sensing matrix $\boldsymbol{\Phi}$.

In the context of the EEG signal, the Gaussian random matrix is commonly used as a CS signal sensing matrix in the literature. Many studies $[31,36,38]$ used the Gaussian random matrix $\boldsymbol{\Phi}$ in their work. In the study proposed by J. Zhu et al. [60] a new algorithm towards CS reconstruction of multichannel EEG signals by exploiting cosparsity and the low-rank property also used Gaussian random matrix as a sensing matrix. Recently, S. Qiu et al. [61] proposed a CS framework for BCIs based on teleoperation control of robotic exoskeleton using steady-state visual evoked potentials (SSVEP). They used Gaussian random matrix in their integrated study of CS, various brain-machine reference commands, and adaptive fuzzy controllers together. The study proposed by $\mathrm{H}$. Liu et al. [62] discussed various random matrices including Gaussian random matrix for resting-state EEG signal classification of schizophrenia. Although Gaussian random matrix is commonly used for measurement sensing, it is not suitable for wireless neural engineering applications because a Gaussian random generator would lead to a large number of matrix-vector multiplications (energy-intensive), and therefore, it cannot be efficiently implemented on a signal acquisition hardware [63]. In order to make the sensing process faster and less computationally complex, few studies $[29,35]$ used a full Bernouilli matrix, as it is easier to generate its random entries with fewer multiplication operations. However, it is still a challenge to use Bernouilli matrix due to its space power consumption in hardware. An alternative to this problem could be the sparse binary sensing matrices, which only contain a few nonzero (one's) entries in each column of the matrix. An interesting study proposed by J. Sheng et al. [64] compared the performance of the random Bernouilli matrix and sparse binary sensing matrix and concluded that sparse binary sensing matrix are often a better choice over random Bernouilli matrix for implementing CS for the wireless body sensor network (WBSN). A comparative performance evaluation study 
proposed by R. Tello et al. [65] on an independent SSVEP-BCI based on CS also used a random sensing matrix in their experiment. This study focuses on the detection of visual attention in SSVEP-BCIs for people with severe motor disabilities. In order to reduce the matrix multiplication operation further, A. Gilbert et al. [66] also used a sparse binary sensing matrix. Although the reconstruction performance of sparse binary sensing matrices is not good enough in comparison to full random matrices, it has been used in many other studies such as $[36,39,51,63,67,68]$. N. Mammone et al. [36] adopted the sparse binary matrix as a measurement matrix, inverse DCT matrix as a dictionary matrix to compress and, subsequently, reconstruct the high-definition (HD)-EEG signals obtained from AD, MCI and healthy subjects. The work proposed by M. Fira et al. [54] also used a sparse binary sensing matrix in their experiments. They also compared the reconstruction error for four different sensing matrices, which are: Gaussian random matrix, Bernouilli random matrix, a random sparse binary sensing matrix, and a fixed sparse binary sensing matrix. In conclusion, they recommended using a sparse binary sensing matrix for future wireless BCI applications. Furthermore, M. Hanafy et al. [69] proposed a study for ZigBee-based EEG telemonitoring and used a binary sensing matrix in their design. Similarly, A. Majumdar et al. [70] also suggested the use of binary sensing matrix to reduce the sensing power consumption in their energy efficient CS-based EEG sensing design.

Numerous research has demonstrated the effectiveness of the aforementioned random sensing matrices. However, the random sensing matrices have high reconstruction complexity, and computationally demanding, which need significant space requirement for storage. In order to go beyond these limitations, there is a growing interest for developing deterministic sensing matrices. T. Nguyen et al. [71] investigated and presented a good review of various deterministic sensing matrices. Furthermore, few research, as H. Monajemi et al. [72], also claimed that randomness of the matrix $\boldsymbol{\Phi}$, such as Gaussian random matrix, is not required for the phase transition phenomenon with the assumption that the positions of the non-zeros are chosen purely at random. The system complexity of the random dense matrices-based approach is typically $\mathbf{O}\left(N^{2}\right)$, whereas implicit operations often can be carried out with the system complexity of $\mathbf{O}(N \log (N))$ using such deterministic sensing matrices [72]. These deterministic sensing matrices do not require explicit data saving space and memory accesses and, therefore, these can be used for designing future highly miniaturized wearable BCIs.

As discussed, the deterministic sensing matrix can make the hardware realization convenient and easy for the considered applications, hence, deterministic sensing has been widely investigated in the literature and already been used in many other applications [73,74]. The deterministic sensing matrix has been exploited for BCI application. For example, W. Zhao et al. [75] used deterministic quasi-cyclic array code (QCAC) matrix-based compressed sensing encoder architecture for wireless neural recording applications. Further, W. Zhao et al. [76] also proposed the construction of the QCAC matrix and sparse random binary matrix (SRBM) and performed simulation experiments to reconstruct EEG signal from compressed measurement. The authors also compared the reconstruction performance of QCAC, SRBM, and random binary matrix and showed that these matrices are energy-efficient and had considerable reduction in computational resources.

\subsection{Reconstruction Algorithms}

It is interesting to note that the signal acquisition, compression and transmission of CS measurements are relatively easy and it is the exact reconstruction of the signal that is complex. On the contrary, it is reverse for the classical sampling based on the Nyquist theorem. A class of CS reconstruction algorithms has been developed and applied to recover the original signal from the compressed measurement of $\mathbf{y}$. The reconstruction algorithms available in the literature can be broadly divided into six categories, as shown in Figure 3. At the receiver end, the CS reconstruction algorithms basically seek to find an exact solution of an underdetermined system of (1) from infinitely many solutions. These algorithms are usually iterative and pursue a key goal to reduce the error between the original and the recovered signal. 


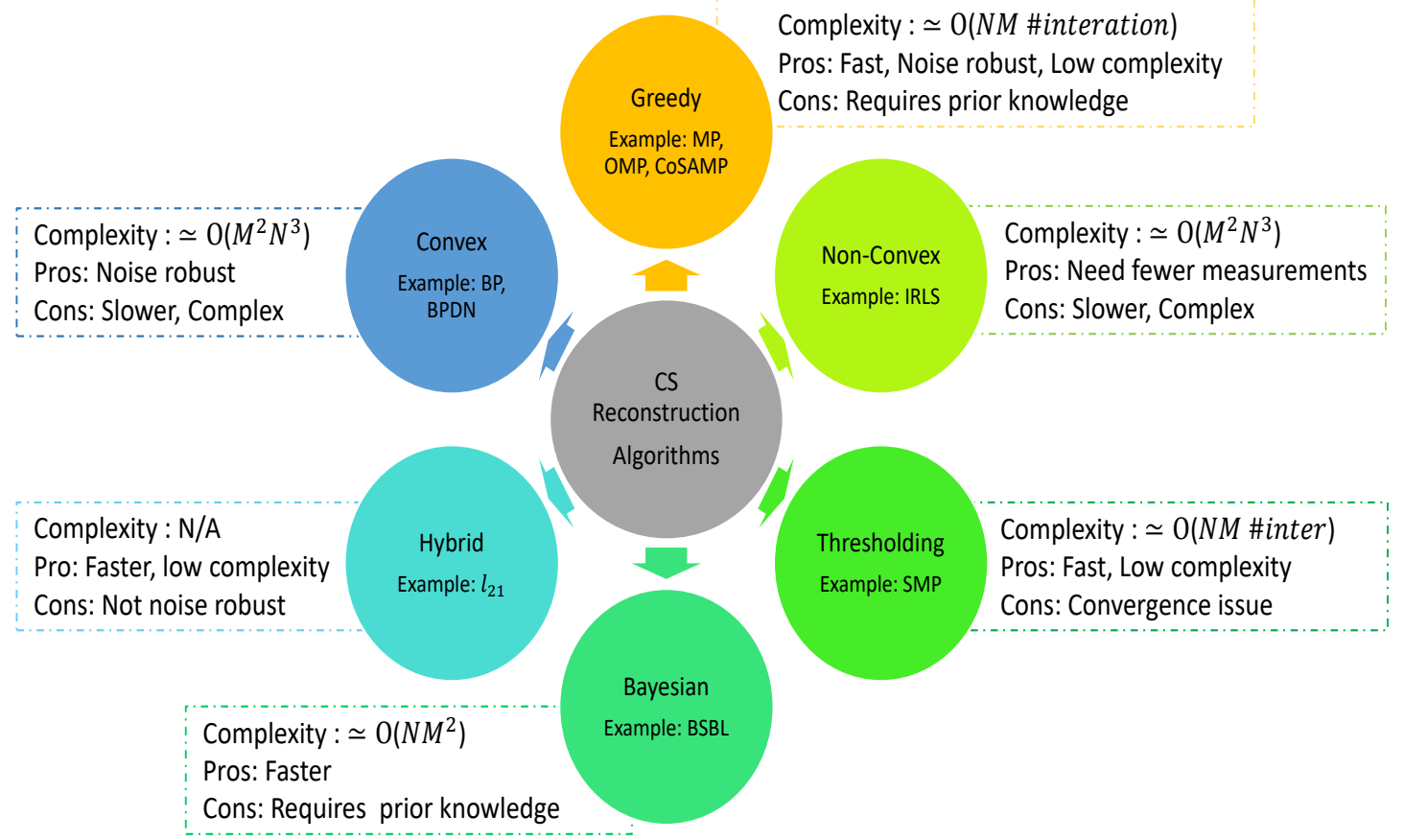

Figure 3. Classification of reconstruction algorithms.

In the context of EEG signal for BCI application, mostly nonlinear algorithms are used, which require prior knowledge of sparsifying $\mathbf{\Psi}$ and $\mathbf{A}$. Basically, the recovery of $\mathbf{s}$ in (3) from $M$ compressed measurements, seeks for solution $\widehat{s}$ by finding minimum few non-zero entries for an under-determined system $N>>M$. Mathematically, the recovery of $\mathbf{s}$ using $l_{0}$-minimization is formulated as:

$$
\widehat{\mathbf{s}}=\underset{\widehat{\mathbf{s}}}{\arg \min }\|\widehat{\mathbf{s}}\|_{1_{0}} \text { subject to }: \mathbf{y}=\mathbf{A} \widehat{\mathbf{s}},
$$

where $\|\widehat{\mathbf{s}}\|_{1_{0}}$ is the number of non-zero entries in s. A limitation of (5) is that it is NP-hard and ill-conditioned because of the non-convex nature of $l_{0}$-minimization. However, (5) can be reformulated into a convex problem and a unique solution can be found by using $l_{1}$-minimization given by (6)

$$
\widehat{\mathbf{s}}=\underset{\widehat{\mathbf{s}}}{\arg \min }\|\widehat{\mathbf{s}}\|_{\mathbf{1}_{1}} \text { subject to }: \mathbf{y}=\mathbf{A} \widehat{\mathbf{s}},
$$

where $\|\widehat{\mathbf{s}}\|_{1_{1}}=\sum\left|\mathbf{s}_{\mathbf{i}}\right|$.

The advantages of $l_{1}$-minimization is its flexibility to incorporate prior information into signal reconstruction model and its uniform recoverability. A convex optimization problem that finds a solution having a minimum $l_{1}$-norm is often called basis pursuit (BP), which is a commonly used approach for signal reconstruction. For instance, F. Morabito et al. [37] used $l_{1}$-norm and proposed an interesting study on finding the compressibility property of EEG signal collected from AD patients, MCI subjects and healthy elderly. Similarly, M. Fira et al. [54] used $l_{1}$-norm for EEG signal reconstruction for the analysis of the specific dictionaries of EEG signals. Furthermore, M. Fira et al. [77] also used $l_{1}$-norm in their study and proposed CS based framework for the P300 detection spelling paradigm.

The $l_{l}$-minimization techniques are powerful methods for CS signal recovery when dealing with noise-free measurements. However, in the case of noisy measurements, it may show a poor recovery performance and this issue can be handled using $l_{2}$ norms. Similar to $l_{1}$ norm, $l_{2}$ norm optimization can be formulated as

$$
\widehat{\mathbf{s}}=\underset{\widehat{\mathbf{s}}}{\arg \min }\|\widehat{\mathbf{s}}\|_{\mathbf{1}_{2}} \text { subject to }: \mathbf{y}=\mathbf{A} \widehat{\mathbf{s}},
$$

where $\|\widehat{\mathbf{s}}\|_{1_{2}}=\sum\left|\mathbf{s}_{\mathbf{i}}{ }^{2}\right|^{1 / 2}$. 
In BCI research, few studies adopted $l_{2}$ norm approach to reconstruct the EEG signal. For example, the study proposed by T. Moy et al. [30] for acquisition and biomarker-extraction system used $l_{2}$ norm for EEG signal reconstruction. Another work proposed by K. Abualsaud et al. [32] also used $l_{2}$ norm for EEG-based epileptic seizure detection. In most BCI applications, $l_{1}$ and $l_{2}$ based approach showed promising results. However, these norms suffer from significant limitations, as they do not take temporal dynamics of the EEG signal into account, they fail to recover the time courses of EEG data. In order to address this limitation, few researchers such as A. Gramfort et al. [78] used combinatorial optimization or mixed norm of both $l_{1}$ and $l_{2}$, also called $l_{21}$ norm optimization for Magneto-EEG signal. Similar to [78], $l_{0}$ norm can be used with other norms to efficiently solve the optimization. For example, Y. Liu et al. [79] used a combinatorial optimization with the $l_{0}$ norm and Schatten ${ }_{0}\left(s_{0}\right)$ norms to encourage cosparsity and low-rank structure in the reconstructed EEG signals. Further, the alternating direction method of multipliers (ADMM) is used to solve the simultaneous cosparsity and low-rank (SCLR) optimization. The study proposed by J. Zhu et al. [60] also proposed an algorithm towards CS reconstruction of multichannel EEG signals via $l_{q}$ norm and $s_{p}$ norm regularization. They make use of $l_{q}$ norm and $s_{p}$ norm to enforce cosparsity and low-rank property in the reconstructed EEG signal. Further, they applied ADMM to the resulting nonconvex optimization and compared the performance of $l_{q}$ norm and $s_{p}$ with other competitive reconstruction algorithms and claimed superior performance.

Furthermore, M. Tayyib et al. [80] proposed an accelerated sparsity-based reconstruction of compressively sensed multichannel EEG signals using ADMM. First, they obtained the $s_{p}$ norm along with the decorrelation transformation of EEG data, and then double temporal sparsity-based reconstruction algorithm has been applied for the signal reconstruction. Few other recent studies [81] have also shown that combining cosparsity and low-rank property usually results in efficient CS reconstruction of multichannel EEG signals. However, these studies rarely incorporated the effect of noise in their studies. It is worth commenting that the noisy measurements may degrade the performance of CS reconstruction algorithms. The study discussed by X. Zouab et al. [82] proposed a robust CS reconstruction algorithm called regularized cosparsity and low-rank property (RCS-CLR) to accurately recover multichannel EEG signals from noisy measurements in the presence of impulsive noise. Furthermore, most of the cosparsity based methods ignore the adjacent relationship between the real physical electrodes and use convex regularizations to exploit cosparsity and channel correlation. This may also degrade the performance of the reconstruction method. In order to enforce inherent correlation across different channels and cosparsity of multichannel EEG signals, X. Zou et al. [81] proposed a graph Fourier transform and nonconvex optimization (GFTN)-based method, which can exploit the accurate adjacent relationship between the real physical channels. Similar to [80], this work also used ADMM for signal reconstruction.

The Bayesian-based method is another commonly used approach for signal reconstruction from compressed measurements. The block sparse Bayesian learning (BSBL) was initially introduced by Z. Zhang et al. [51] as an alternative CS reconstruction algorithm and achieved promising results for telemonitoring of nonsparse signals such as EEG. The work proposed by K. Zeng et al. [29] and N. Mammone et al. [36] also adopted the BSBL algorithm as EEG reconstruction method. The work proposed by S. Fauvel et al. [63] exploits both temporal and spatial correlations to efficiently compress EEG signals in WBSNs and used BSBL based approach for EEG reconstruction. In this study, the authors compared the energy consumption (for cycle count, run time, computation and computation plus transmission) and reconstruction accuracy of the various framework on a wide range of EEG applications. The BSBL algorithm showed satisfactory reconstruction performance for various applications. Particularly, for reconstruction of multichannel EEG signals, it is time-consuming. To address this issue, Z. Zhang et al. [83] proposed a spatiotemporal sparse Bayesian learning algorithm to reconstruct multichannel signals simultaneously for BCIs and EEG-based driver's drowsiness detection by exploiting temporal correlation within each channel and inter-channel correlation among different channel signals. Furthermore, H. Mahrous et al. [68] proposed a novel method 
for compressing multi-channel EEG signals by exploiting both linear and non-linear dependencies in the EEG data. This study enables the conventional BSBL-BO to produce a better reconstruction of the EEG signal because of significantly better "block" sparsity structure than the state-of-art BSBL-BO. B. Liua et al. [84] proposed a fast BSBL (BSBL-FM) reconstruction algorithm CS framework. They also implemented BSBL in FPGA and compared the performance of CS-based and wavelet-based compression algorithms in terms of power and energy consumption.

As discussed before, BP uses $l_{1}$ norm minimization to solve the optimization problem. Greedy algorithms pursue a similar goal as convex algorithms to minimize the error between the original signal and the recovered signal. In the BCI literature, many variations of BP are proposed. For example, $\mathrm{H}$. Lee et al. [41] presented an automatic sleep-stage classification system and used the orthogonal matching pursuit (OMP) algorithm for EEG signal reconstruction. Similarly, B. Kaliannan et al. [38] proposed a hardware realization of the multichannel EEG signal based on simultaneous orthogonal matching pursuit (SOMP) and theory of joint sparse recovery for EEG reconstruction. The study discussed in [85] also utilized basic SOMP along with other algorithms for distributed CS of jointly sparse signals. Furthermore, in order to deal with non-sparsity on the EEG signal, H. Djelouat et al. [86] used subspace pursuit algorithm with the concept of a concatenated basis where a sparsifying basis consists of randomly selected atoms from both DCT and DWT. The work proposed by R. Kus et al. [47] provides more mathematical details on OMP properties and its advantages, and proposed multivariate matching pursuit for EEG signal reconstruction. Furthermore, basis pursuit denoising (BPDN) is an another computationally efficient approach used in the BCI literature to reconstruct the EEG signal. For instance, the study proposed in M. Hosseini et al. [39] have used BPDN with a joint sparse decoding algorithm for reconstruction of multichannel intracranial neural data and X. Li et al. [67] used BPDN to understand the effect of epoch length on CS reconstruction. In the literature, various other reconstruction algorithms are also proposed for specific applications, such as A. Khoshnevis et al. [87], which used a novel reconstruction method for event-related potential signals using Kronecker approach to improve the quality of reconstruction and accelerate the compression phase. In the study proposed by R. Shriwastav et al. [40], the convolutional neural network (CNN) is used to reconstruct the motor imagery EEG signal.

In addition to the aforementioned studies, few research has been focused on comparing the reconstruction performance of various approaches proposed. For example, X. Zouab et al. [82] compared the performance of RCS-CLR with state-of-the-art methods such as ADMM-based SCLR (SCLR-A) [78], BSBL [51], and SOMP [38] and showed a superior reconstruction performance in the presence of noise. In the study by Z. Zhang et al. [88] reported a comparison result of twelve typical sparse signal recovery algorithms such as compressive sampling matching pursuit (CoSaMP), hard thresholding pursuit (HTP), subspace pursuit, among others for EEG signals. Similarly, A. Abdulghani et al. [31] compared the performance of 18 different combinations of BP, MP, and OMP with six dictionary matrices (Gabor, Mexican hat, spline- linear and cubic, and B-spline- linear and cubic) for long term scalp EEG signals. The experiments performed by M. Rani et al. [89] compared the performance of the CS reconstruction algorithms such as BP, BPDN, OMP, and CoSaMP for determining a better reconstruction of EEG signal, and concluded that if reconstruction speed is a prime concern, then OMP is a better choice, whereas, for higher reconstruction quality, CoSaMP is a preferred option. Furthermore, R. Tello et al. also compared the classification performance of EEG signal reconstructed using three algorithms namely BSBL-BO, $l_{p}^{d}$ - regularized least-squares $\left(l_{p}^{d}\right.$-RLS) and $l_{p}^{2 d}$-RLS. The results in this study reflect that $l_{p}$-RLS based reconstruction approach shows better performance compared to the BSBL-BO. The effectiveness of the improved $l_{p}$-RLS reconstruction approach is also demonstrated by D. Gurve et al. [90] for other applications such as fetal electrocardiogram (ECG) extraction from abdominal ECG signal.

A summary of the detailed empirical comparison of selected state-of-the-art CS-based BCI methods in terms of reconstruction algorithms, sensing matrix, and sparse basis used is shown in Tables 1 and 2. 
Table 1. A summary of CS applied EEG studies (application focused).

\begin{tabular}{|c|c|c|c|c|}
\hline Studies & Applications & $\begin{array}{l}\text { Reconstruction } \\
\text { Algorithms }\end{array}$ & $\begin{array}{l}\text { Sensing } \\
\text { Matrices }\end{array}$ & $\begin{array}{l}\text { Sparse } \\
\text { Basis }\end{array}$ \\
\hline $\begin{array}{l}\text { A. Abdulghani et al. } \\
{[31], 2012}\end{array}$ & $\begin{array}{c}\text { Seizure } \\
\text { detection }\end{array}$ & $\begin{array}{c}\mathrm{BP}, \mathrm{MP}, \\
\mathrm{OMP}\end{array}$ & $\begin{array}{l}\text { Gaussian random } \\
\text { matrix }\end{array}$ & $\begin{array}{c}\text { Gabor } \\
\text { dictionary }\end{array}$ \\
\hline $\begin{array}{c}\text { K. Abualsaud et al. } \\
\text { [32], } 2013\end{array}$ & $\begin{array}{c}\text { Seizure } \\
\text { detection }\end{array}$ & $\begin{array}{c}l_{2} \text { norm } \\
\text { minimization }\end{array}$ & $\begin{array}{l}\text { Random } \\
\text { matrix }\end{array}$ & $\begin{array}{c}\text { DCT } \\
\text { dictionary }\end{array}$ \\
\hline $\begin{array}{c}\text { F. Morabito et al. } \\
\text { [37], } 2013\end{array}$ & $\begin{array}{c}\text { Alzheimer's } \\
\text { disease analysis }\end{array}$ & $\begin{array}{c}l_{1} \text { norm } \\
\text { minimization }\end{array}$ & $\begin{array}{c}\text { Gaussian random } \\
\text { matrix }\end{array}$ & $\begin{array}{c}\text { Gabor } \\
\text { dictionary }\end{array}$ \\
\hline $\begin{array}{l}\text { M. Shoaib et al. } \\
\text { [34], } 2014\end{array}$ & $\begin{array}{c}\text { Seizure } \\
\text { detection }\end{array}$ & $\begin{array}{l}\text { Reconstruction } \\
\text { free }\end{array}$ & $\begin{array}{l}\text { Random } \\
\text { matrix }\end{array}$ & - \\
\hline $\begin{array}{l}\text { B. Liua et al. } \\
\text { [84], } 2014\end{array}$ & $\begin{array}{c}\text { Seizure } \\
\text { detection }\end{array}$ & BSBL-FM & $\begin{array}{l}\text { Sparse binary } \\
\text { matrix }\end{array}$ & $\begin{array}{c}\text { Wavelet } \\
\text { dictionary }\end{array}$ \\
\hline $\begin{array}{l}\text { Z. Zhang et al. } \\
\text { [83], } 2014\end{array}$ & $\begin{array}{c}\text { SSVEP \& } \\
\text { Drowsiness detection }\end{array}$ & STSBL-EM & $\begin{array}{l}\text { Sparse binary } \\
\text { matrix }\end{array}$ & $\begin{array}{c}\text { DCT } \\
\text { dictionary }\end{array}$ \\
\hline $\begin{array}{l}\text { K. Zeng et al. } \\
\text { [29], } 2016\end{array}$ & $\begin{array}{c}\text { Seizure } \\
\text { detection }\end{array}$ & BSBL & $\begin{array}{l}\text { Bernoulli random } \\
\text { matrix }\end{array}$ & $\begin{array}{c}\text { Gabor } \\
\text { dictionary }\end{array}$ \\
\hline $\begin{array}{l}\text { M. Fira et al. } \\
\text { [54], } 2016\end{array}$ & $\begin{array}{c}\text { P300 } \\
\text { spelling }\end{array}$ & $\begin{array}{c}l_{1} \text { norm } \\
\text { minimization }\end{array}$ & $\begin{array}{l}\text { Random } \\
\text { matrix }\end{array}$ & $\begin{array}{c}\text { Wavelet } \\
\text { dictionary }\end{array}$ \\
\hline $\begin{array}{l}\text { M. Fira et al. } \\
\text { [77], } 2016\end{array}$ & $\begin{array}{c}\text { P300 } \\
\text { spelling }\end{array}$ & $\begin{array}{c}l_{1} \text { norm } \\
\text { minimization }\end{array}$ & $\begin{array}{l}\text { Random } \\
\text { matrix }\end{array}$ & $\begin{array}{l}\text { Data driven } \\
\text { dictionary }\end{array}$ \\
\hline $\begin{array}{c}\text { M. Shoaran et al. } \\
\text { [35], } 2016\end{array}$ & $\begin{array}{c}\text { Seizure } \\
\text { detection }\end{array}$ & $\begin{array}{l}\text { Reconstruction } \\
\text { free }\end{array}$ & $\begin{array}{c}\text { Bernoulli binary } \\
\text { matrix }\end{array}$ & - \\
\hline $\begin{array}{l}\text { T. Moy et al. } \\
\text { [30], } 2017\end{array}$ & $\begin{array}{c}\text { Seizure } \\
\text { detection }\end{array}$ & $\begin{array}{c}l_{1} \text { norm } \\
\text { minimization }\end{array}$ & $\begin{array}{l}\text { Random } \\
\text { matrix }\end{array}$ & $\begin{array}{c}\text { Gabor } \\
\text { dictionary }\end{array}$ \\
\hline $\begin{array}{l}\text { R. Aghazadeh et al. } \\
\text { [33], } 2018\end{array}$ & $\begin{array}{c}\text { Seizure } \\
\text { detection }\end{array}$ & $\begin{array}{c}\text { Reconstruction } \\
\text { free }\end{array}$ & - & - \\
\hline $\begin{array}{l}\text { H. Lee et al. } \\
\text { [41], } 2019\end{array}$ & $\begin{array}{l}\text { Sleep-Stage } \\
\text { Classification }\end{array}$ & OMP & $\begin{array}{c}\text { Random binary } \\
\text { matrix }\end{array}$ & - \\
\hline $\begin{array}{l}\text { N. Mammone et al. } \\
{[36], 2019}\end{array}$ & $\begin{array}{c}\text { Alzheimer's } \\
\text { disease analysis }\end{array}$ & BSBL & $\begin{array}{l}\text { Sparse binary } \\
\text { matrix }\end{array}$ & $\begin{array}{c}\text { DCT } \\
\text { transform }\end{array}$ \\
\hline $\begin{array}{c}\text { R. Shrivastwa et al. } \\
{[40], 2020}\end{array}$ & $\begin{array}{l}\text { Motor } \\
\text { imagery }\end{array}$ & CNN-Based & $\begin{array}{c}\text { Bernoulli } \\
\text { random matrix }\end{array}$ & - \\
\hline \multicolumn{5}{|c|}{$\begin{array}{l}\text { Glossary of terms: Alternating Direction Method of Multipliers (ADMM); Basic Pursuit (BP); Basis Pursuit } \\
\text { Denoising (BPDN); Block Sparse Bayesian Learning (BSBL); Block Sparse Bayesian Learning-Bounded } \\
\text { Optimization (BSBL-BO); Block Sparse Bayesian Learning-Fast Marginalized (BSBL-FM); Compressive } \\
\text { Sampling Matching Pursuit (CoSaMP); Graph Fourier Transform and Nonconvex (GFTN); Hard Thresholding } \\
\text { Pursuit (HTP); Iteratively Reweighted Least Square (IRLS); Orthogonal Matching Pursuit (OMP); Regularized } \\
\text { Cosparsity and Low-Rank (RCS-CLR); Regularized Least-Squares (RLS); Simultaneous Orthogonal Matching } \\
\text { Pursuit (SOMP). }\end{array}$} \\
\hline
\end{tabular}


Table 2. A summary of CS applied EEG studies (signal reconstruction focused).

\begin{tabular}{|c|c|c|c|}
\hline Studies & $\begin{array}{l}\text { Reconstruction } \\
\text { Algorithm }\end{array}$ & $\begin{array}{l}\text { Sensing } \\
\text { Matrix }\end{array}$ & $\begin{array}{l}\text { Sparse } \\
\text { Basis }\end{array}$ \\
\hline $\begin{array}{l}\text { M. Hosseini et al. } \\
\text { [39], } 2013\end{array}$ & $\mathrm{BPDN}$ & $\begin{array}{l}\text { Sparse binary } \\
\text { matrix }\end{array}$ & $\begin{array}{l}\text { Gabor } \\
\text { dictionary }\end{array}$ \\
\hline $\begin{array}{l}\text { Z. Zhang et al. } \\
\text { [51], } 2013\end{array}$ & BSBL-BO & $\begin{array}{l}\text { Sparse binary } \\
\text { matrix }\end{array}$ & $\begin{array}{c}\text { DCT } \\
\text { dictionary }\end{array}$ \\
\hline $\begin{array}{l}\text { R. Kus et al. } \\
{[47], 2013}\end{array}$ & $\begin{array}{c}\text { Multivariate } \\
\text { matching pursuit }\end{array}$ & $\begin{array}{l}\text { Random } \\
\text { matrix }\end{array}$ & $\begin{array}{l}\text { Gabor } \\
\text { dictionary }\end{array}$ \\
\hline $\begin{array}{l}\text { S. Fauvel et al. } \\
\text { [63], } 2014\end{array}$ & BSBL-BO & $\begin{array}{l}\text { Sparse binary } \\
\text { matrix }\end{array}$ & $\begin{array}{c}\text { Gabor } \\
\text { dictionary }\end{array}$ \\
\hline $\begin{array}{l}\text { Y. Liu et al. } \\
\text { [79], } 2015\end{array}$ & $\begin{array}{l}l_{0} \text { norm } \\
\text { and } S_{0} \text { norm }\end{array}$ & $\begin{array}{c}2^{\text {nd }} \text { order } \\
\text { difference matrix }\end{array}$ & - \\
\hline $\begin{array}{l}\text { B. Kaliannan et al. } \\
\text { [38], } 2016\end{array}$ & DCS-SOMP & $\begin{array}{c}\text { Gaussian random } \\
\text { matrix }\end{array}$ & $\begin{array}{c}\text { Joint } \\
\text { sparsity }\end{array}$ \\
\hline $\begin{array}{l}\text { H. Mahrous et al. } \\
\text { [68], } 2016\end{array}$ & BSBL-BO & $\begin{array}{l}\text { Sparse binary } \\
\text { matrix }\end{array}$ & $\begin{array}{c}\text { DCT } \\
\text { dictionary }\end{array}$ \\
\hline $\begin{array}{l}\text { J. Zhu et al. } \\
{[60], 2016}\end{array}$ & $\begin{array}{c}l_{q} \text { norm } \\
\text { and } S_{q} \text { norm }\end{array}$ & $\begin{array}{l}\text { Gaussian random } \\
\text { matrix }\end{array}$ & $\begin{array}{c}\text { Wavelet } \\
\text { dictionary }\end{array}$ \\
\hline $\begin{array}{l}\text { H. Djelouat et al. } \\
\text { [86], } 2017\end{array}$ & $\begin{array}{l}\text { Subspace } \\
\text { pursuit }\end{array}$ & $\begin{array}{l}\text { Bernoulli } \\
\text { random matrix }\end{array}$ & $\begin{array}{l}\text { Wavelet } \\
\text { transform }\end{array}$ \\
\hline $\begin{array}{l}\text { X. Li et al. } \\
\text { [67], } 2018\end{array}$ & $\mathrm{BPDN}$ & $\begin{array}{l}\text { Sparse binary } \\
\text { matrix }\end{array}$ & $\begin{array}{l}\text { Gabor } \\
\text { dictionary }\end{array}$ \\
\hline $\begin{array}{l}\text { S. Khoshnevis et al. } \\
\text { [87], } 2019\end{array}$ & $\begin{array}{l}\text { Kronecker-based } \\
\text { technique }\end{array}$ & $\begin{array}{c}\text { Deterministic binary } \\
\text { matrix }\end{array}$ & - \\
\hline $\begin{array}{l}\text { M. Rani et al. } \\
\text { [89], } 2019\end{array}$ & $\begin{array}{c}\text { BP, BPDN, } \\
\text { OMP,CoSaMP }\end{array}$ & $\begin{array}{l}\text { pseudorandom } \\
\text { sequence }\end{array}$ & $\begin{array}{l}\text { Fourier } \\
\text { transform }\end{array}$ \\
\hline $\begin{array}{l}\text { M. Tayyib et al. } \\
\text { [80], } 2020\end{array}$ & ADMM & $\begin{array}{c}\text { sparse } \\
\text { circulant matrix }\end{array}$ & $\begin{array}{l}\text { Fourier } \\
\text { transform }\end{array}$ \\
\hline $\begin{array}{l}\text { X. Zou et al. } \\
\text { [81], } 2020\end{array}$ & ADMM & $\begin{array}{c}2^{\text {nd }} \text { order } \\
\text { difference matrix }\end{array}$ & $\begin{array}{c}\text { Graph } \\
\text { Fourier transform }\end{array}$ \\
\hline
\end{tabular}

\section{Reconstruction Free CS}

As discussed in earlier sections, a number of CS algorithms are being actively pursued in the field of neural engineering, however, to satisfy the constraints of many practical BCI applications it is essential to minimize the computational complexity of the CS reconstruction algorithm. In contrast, the high computational complexity of available CS reconstruction algorithms is an obstacle for many real-time applications. For instance, for basis pursuit and CoSAMP, the computational complexity is $\mathbf{O}\left(N^{3}\right)$ and $\mathbf{O}(M, N)$, respectively. Few studies [91-93] discussed on the computational complexity of various other $\mathrm{CS}$ reconstruction algorithms in detail.

Interestingly, for some classification applications, we may not require an accurate reconstruction signal of interest to extract the features for classification. In these cases, it may be possible to perform feature extraction and classification directly on the compressed measurements, without reconstructing the signal of interest. More specifically, different from the previous traditional CS, the signal of interest can be compressed using CS at the sensing node and the features can be extracted directly from compressed measurements at the receiver end. This reconstruction free CS approach is called "compressive feature learning". Figure 4 shows the reconstruction-free compressive learning CS framework. In this section, we take an in-depth look at the currently available reconstruction free CS 
approaches in the developing field of BCIs from the perspective of the machine learning classification and system complexity.
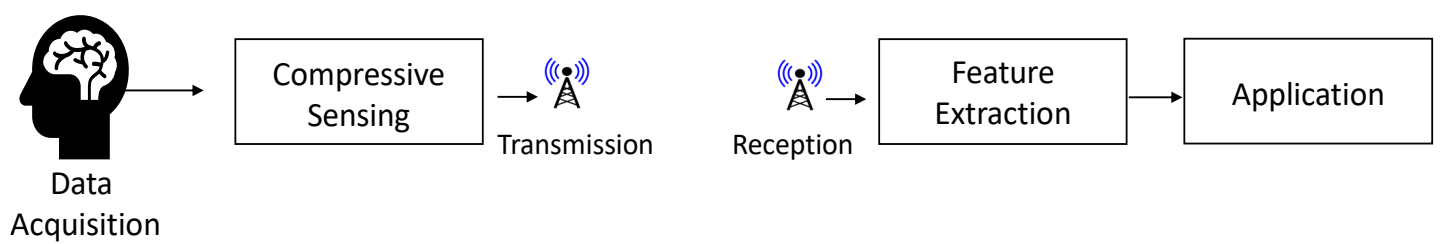

Figure 4. Block diagram of reconstruction free CS-based EEG studies.

Motivated by the fact that meaningful features can be extracted from the compressed measurement directly, many researchers have focused on reconstruction-free compressive learning for various applications such as surveillance and autonomous navigation [94], compressive cameras [95], and text data classification [96]. Furthermore, Lohit et al. [97,98] and Braun et al. [99] also hypothesize that the reconstruction step can be entirely bypassed, and shown the optimal machine learning performance for computer vision applications, using reconstruction-free compressive learning. In the context of neural engineering, very limited but successful attempts have been made towards the feature extraction from the compressed domain. For instance, M. Shoaib et al. [34] proposed a seizure-detection system that directly uses compressively sensed EEG measurements for embedded signal analysis. In particular, they proposed an algorithm and a hardware architecture that enables two power-management knobs to quantify the amount of data compression and to determine the approximation error for the compressed-domain analysis. Similarly, M. Shoaran et al. [35] proposed a multichannel compressed-domain feature extraction as a low-power technique for data compression and seizure detection from multichannel cortical implants. This work also shows the success of using compressed feature learning, which reduces the computational energy for analysis due to a reduction in reconstruction costs. In order to leverage the benefits of compressed-domain feature extraction, R. Aghazadeh et al. [14] also proposed a new algorithm for epileptic seizure detection using a compressively sensed multichannel EEG signal. S. Qiu et al. [61] utilized the concept of feature extraction from the compressed measurements and proposed a teleoperation control system for robotic exoskeleton performing manipulation tasks. This work conveys the control signals to the robotic exoskeleton using features extracted directly from compressed SSVEP measurements. Furthermore, M. Fira et al. [100] also proposed EEG signals classification for a spelling paradigm in the compressed domain.

In summary, the main advantage of the reconstruction free CS approach is that with the feature extraction from compressed measurements directly, the reconstruction step at the receiver node can be entirely bypassed and, therefore, greatly reduces the computation complexity of the system. Additionally, the reconstruction free CS approach not only reduces the system complexity but also reduces the processing time at the receiver end. On the other hand, the reconstruction free CS is an analysis framework and signal synthesis is not a desired outcome.

\section{Discussion and Future Directions}

Neuroscience is a fascinating field and has a profound impact on people's health. The possible technologies that to be integrated with future neural interface technology certainly have the potential to improve our health even more. We are probably standing on the threshold of a complete transformation of the neuroscience industry by the use of big brain data from clinical and consumer-directed neurotechnological devices, which may provide reduced latency and permit a real bidirectional interaction with our brain. Furthermore, the applications of neural implants in the current neuroscience industry are still in their infancy, but we are likely to see in the future functionalities of a neural interface, starting from brain-to-brain connectivity, cell level the neural activation of the different 
channels, personalized treatments in patients affected by chronic neurological diseases to monitor the production of neurotransmitters, direct stimulation of neurons and bidirectional brain-to-machine interoperability in the forthcoming years.

The CS-based wireless neurotechnological devices can address the challenges encountered in a low power application where sensing power is very critical such as battery-operated EEG sensing implants. However, in CS for neurological signals, there is no "bread and butter" solution, which means no one algorithm works well for every problem and cannot be assured for all the applications. This is widely applicable at the receiver end of CS where we reconstruct the original signal using various algorithms. As a result, one should use a different algorithm for different applications, which would lead to optimum results based on the nature of the signal used in the BCI framework. However, in a more practical way, it can be concluded that BSBL and BPDN are a more suitable choice for most applications, where accurate reconstruction is needed, and computation time is not of primary concern. However, for most of the real-time applications, thresholding-based reconstruction could help where the computation time is not of primary concern. Furthermore, BPDN outperforms OMP and BSBL in case of noisy measurements for accurate reconstruction. A similar conclusion can be drawn for the BCIs where computational complexity is a design concern. In such case, reconstruction algorithms such as OMP is preferable. However, the performance of any reconstruction algorithms depends on many other parameters. For instance, X. Li et al. [67] investigated the effect of EEG signals epoch length on the CS reconstruction algorithm and concluded that a longer epoch length leads to better signal compression at the expense of larger signal reconstruction time. The findings in this paper shows that at the sampling frequency of $256 \mathrm{~Hz}$, a 4-s epoch length is suitable to perform the signal reconstruction. However, the signal reconstruction performance also depends on the performing hardware platform not just on the epoch length of an EEG signal. Furthermore, apart from the accurate reconstruction of the EEG signal, the accurate identification of single-unit neural activities (spikes) has a key role in developing high-accuracy BCIs. To this end, various works have been done such as dynamic evolving SSNs [101] and spiking neural networks (SNNs) [102] and demonstrated that an SSN does a strong compression of the EEG signal when it is coded as spikes. In context of CS for BCIs, [40] also showed that spike signals are highly compressed using the CS technique with up to a $90 \%$ compression rate.

Recent progress has suggested how CS can help to optimize sensing resources, transmission, and storage capacities, as well as to facilitate signal processing in an energy constraint BCI environment. Despite the attention that CS has received in recent years in various fields of neural engineering, few limitations still reside. We conclude this review by raising the following five major challenges and opportunities to be addressed in the future of big data processing, which will be aided by further developments in the CS field.

- Despite of recent advances in hardware technologies and the feasibility of performing on-chip signal processing, most of $\mathrm{BCI}$ research reviewed in this article demonstrated the applicability of CS framework with digital implementation, which relies on many assumptions. In the future, it is expected to see more analog implementations of CS to minimize energy consumption under real-life conditions with built-in hardware.

- Although random matrices are widely used for CS-based BCIs and ensure high reconstruction accuracy, storing random matrices requires a lot of memory. This disadvantage of the random sensing matrix can be addressed through their deterministic construction. The advantages of the deterministic matrix are simplicity in sampling and reconstruction stages, and reduced computational complexity. In the future, it would also be interesting to develop strategies for CS measurements under a deterministic sensing matrix for wireless BCIs.

- In the study proposed by F. Chen et al. [103], a hardware-efficient circuit model was developed for the analysis of analog and digital implementations of the CS-based EEG compression model. The analysis in this study reveals that a digital implementation of CS is a significantly more energy-efficient and suitable architecture with respect to their proposed analog implementation. 
In future research, an efficient analog implementation of CS needs to be explored more for next-generation CS-BCI applications.

- The advancements in the acquisition and processing of large data sets in the field of neural engineering is permitting a greater understanding and clinical observations of patients with brain disease. However, most of the current research has not yet achieved a personalized data-driven approach for treatment. In other words, the current research lacks in terms of developing a quantitative integrative tool to translate these understanding and clinical observations to the individual level to build the basis for personalized treatment. This is an important area which should be considered for future studies.

- The CS and BCI systems have created a hope for the implementation of many practical applications, but as data volumes go up, and BCI research are moving towards the creation of computationally demanding algorithms for more complex applications, CS would not help for real-time applications. In such case, reconstruction free learning could help for many machine learning-based applications. In future, such reconstruction free learning will be more highlighted for many other BCI applications.

The CS is an energy-efficient approach for future BCIs application, which has been developed over the past few years. However, there are still numerous BCIs application areas in which the CS theory continues to be developed including rehabilitation and restoration [104,105], communication and control [106,107], prevention [108,109], and user state monitoring [110,111]. Although it is beyond the scope of this particular review article, security $[112,113]$ and entertainment $[114,115]$ are other very interesting topics in the BCI field, which can be benefited with CS and should be considered for future studies.

\section{Conclusions}

The CS framework can help in dealing with many challenges that current BCIs may encounter, which requires the use of fast, long-term, and energy-saving computational approaches. In this article, we have reviewed the available BCIs, which have benefited from the theory of CS in terms of sparse signal representation, sensing matrix, and available reconstruction methods. The discussion around multiple CS reconstruction algorithms reviewed in this article gives us a more complete landscape of this problem as different algorithms come with different trade-offs, such as time and computational complexity. We have also highlighted the advantages and disadvantages of the available algorithms to explore these trade-offs. Though the main focus of this article is to study CS and related BCIs available so far, the paper nevertheless also discusses the idea of reconstruction free CS used for various applications to reduce the complexity of the system.

Author Contributions: Conceptualization, S.K., D.G.; methodology, S.K., D.G., D.D.-R., and T.B.; software, D.G., S.K.; validation, D.G., D.D.-R., S.K., and T.B.; formal analysis, D.G., S.K., D.D.-R., and T.B.; investigation, D.G., D.D.-R., T.B., and S.K.; resources, D.G., S.K.; data curation, D.G., D.D.-R., T.B., and S.K.; writing一original draft preparation, D.G.; writing-review, and editing, D.G., D.D.-R., T.B., and S.K.; visualization, D.G.; supervision, S.K., and T.B.; project administration, S.K., and T.B.; funding acquisition, S.K. All authors have read and agreed to the published version of the manuscript.

Funding: This research was funded by Natural Sciences and Engineering Research Council of Canada (NSERC) Discovery Grant grant number RGPIN 2020-04628.

Acknowledgments: The authors would like to thank the Natural Sciences and Engineering Research Council of Canada (NSERC) for providing the funding support.

Conflicts of Interest: The authors declare no conflict of interest.

\section{References}

1. Van, H.; Toga A. Human Neuroimaging as a "Big Data" Science. Brain Imaging Behav. 2014, 8, 323-331.

2. Musk, E. An integrated brain-machine interface platform with thousands of channels. J. Med. Int. Res. 2019, 21, e16194. [CrossRef] [PubMed] 
3. Landhuis, E. Neuroscience: Big brain, big data. Nature 2017, 541, 559-561. [CrossRef] [PubMed]

4. Donoho, D. Compressed sensing. IEEE Trans. Inf. Theory 2006, 52, 1289-1306. [CrossRef]

5. Yousufi, M.; Amir, M.; Javed, U.; Tayyib, M.; Abdullah, S.; Ullah, H.; Qureshi, I.M.; Alimgeer, K.; Waseem Akram, M.; Khan, K. Application of Compressive Sensing to Ultrasound Images: A Review. BioMed Res. Int. 2019, 2019, 7861651. [CrossRef] [PubMed]

6. Nagesh, P.; Baoxin, L. A compressive sensing approach for expressioninvariant face recognition. In Proceedings of the 2009 IEEE Conference on Computer Vision and Pattern Recognition, Miami, FL, USA, 20-25 June 2009.

7. Qiao, L.; Chen, S.; Tan, X. Sparsity preserving projections with applications to face recognition. Pattern Recognit. 2010, 43, 331-341. [CrossRef]

8. Duarte, M.F.; Davenport, M.A.; Takhar, D.; Laska, J.N.; Sun, T.; Kelly, K.F.; Baraniuk, R.G. Single-pixel imaging via compressive sampling. IEEE Signal Process Mag. 2008, 2, 83-91. [CrossRef]

9. Okada, H.; Shuhei, S.; Tatsuya, K.; Kentaro, K.; Masaaki, K. Link quality information sharing by compressed sensing and compressed transmission for arbitrary topology wireless mesh networks. IEICE Trans. Commun. 2017, 100, 456-464. [CrossRef]

10. Aderohunmu, F.A.; Brunelli, D.; Deng, J.; Purvis, M. A data acquisition protocol for a reactive wireless sensor network monitoring application. Sensors 2015, 15, 10221-10254. [CrossRef]

11. Sharma, S.; Lagunas, E.; Chatzinotas, S.; Ottersten, B. Application of compressive sensing in cognitive radio communications: A survey. IEEE Commun. Surv. Tutor. 2016, 18, 1838-1860. [CrossRef]

12. Salahdine, F.; Kaaboucha, N.; Ghazib, H. A survey on compressive sensing techniques for cognitive radio networks. Phys. Commun. 2016, 20, 61-73. [CrossRef]

13. Lei, Z.; Yanga, K.; Duan, R.; Xiao, P. Localization of lowfrequency coherent sound sources with compressive beamforming-based passive synthetic aperture. J. Acoust. Soc. Am. 2015, 137, EL255-EL260. [CrossRef] [PubMed]

14. You, H.; Zhixian, M.A.; L.I.W.; Zhu, J. A speech enhancement method based on multi-task Bayesian compressive sensing. IEICE Trans. Inf. Syst. 2017, 100, 556-563. [CrossRef]

15. Giacobello, D.; Christensen, M.G.; Murthi, M.N.; Jensen, S.H.; Moonen, M. Retrieving sparse patterns using a compressed sensing framework: Applications to speech coding based on sparse linear prediction. IEEE Signal Process. Lett. 2010, 17, 103-106. [CrossRef]

16. Huang, Q.; Qu, L.; Wu, B.; Fang, G. UWB through-wall imaging based on compressive sensing. IEEE Trans. Geosci. Remote Sens. 2010, 48, 1408-1415. [CrossRef]

17. Gurbuz, A.; McClell, J.; Waymond, R. Compressive sensing for subsurface imaging using ground penetrating radar. Signal Process. 2009, 89, 1959-1972. [CrossRef]

18. Victor, J.; Kova, B.; Zhou, D.; Cai, D. Efficient image processing via compressive sensing of integrate-and-fire neuronal network dynamics. Neurocomputing 2016, 171, 1313-1322.

19. Ujan, S.; Ghorshi, S.; Pourebrahim, M.; Khoshnevis, S. On the Use of Compressive Sensing for Image Enhancement. In Proceedings of the UKSim-AMSS 18th International Conference on Computer Modelling and Simulation (UKSim), Cambridge, CA, USA, 6-8 April 2016; pp. 167-171.

20. Mun, S.; Fowler, J. Residual reconstruction for block-based compressed sensing of video. In Proceedings of the Data Compression Conference, Snowbird, UT, USA, 29-31 March 2011; pp. 183-192.

21. Veeraraghavan, A.; Reddy, D.; Raskar, R. Coded strobing photography: Compressive sensing of high speed periodic videos. IEEE Trans. Pattern Anal. Mach. Intell. 2011, 33, 671-686. [CrossRef]

22. Barranca, V.; Zhou, D. Compressive sensing inference of neuronal network connectivity in balanced neuronal dynamics. Front. Neurosci. 2019, 13, 1101. [CrossRef]

23. Deka B.; Datta S. Compressed Sensing Magnetic Resonance Image Reconstruction Algorithms; Springer Series on Bio-and Neurosystems; Springer: Singapore, 2019.

24. Kopanoglu, E.; Güngör, A.; Kilic, T.; Saritas, E.; Oguz, K.; Çukur, T.; Güven, H. Simultaneous use of individual and joint regularization terms in compressive sensing: Joint reconstruction of multi-channel multi-contrast MRI acquisitions. NMR Biomed. 2020, 33, e4247. [CrossRef]

25. Meenu, R.; Dhok, S.; Deshmukh, R. EEG Monitoring: Performance Comparison of Compressive Sensing Reconstruction Algorithms. In Information Systems Design and Intelligent Applications; Springer: Singapore, 2019; pp. 9-17. 
26. Mellors, B.; Ben, O.; Mellors, L.; Howle, C.; Spear, M.; Dehghani, H. Compressive Sensing Based Spatial Frequency Domain Imaging Reconstruction. In Proceedings of the Optical Tomography and Spectroscopy 2020, Washington, DC, USA, 20-23 April 2020.

27. Cabral, T.W.; Khosravy, M.; Dias, F.M.; Monteiro, H.L.; Lima, M.A.; Silva, L.R.; Naji, R.; Duque, C.A. Compressive sensing in medical signal processing and imaging systems. In Sensors for Health Monitoring; Academic Press: Cambridge, MA, USA, 2019, pp. 69-92.

28. Meenu, R.; Dhok, S.; Deshmukh, R. EEG Seizure Detection from Compressive Measurements. In Advances in VLSI, Communication, and Signal Processing; Springer: Singapore, 2020; pp. 963-969.

29. Zeng, K.; Yan, J.; Wang, Y.; Sik, A.; Ouyang, G.; Li, X. Automatic detection of absence seizures with compressive sensing EEG. Neurocomputing 2016, 171, 497-502. [CrossRef]

30. Moy, T.; Huang, L.; Rieutort-Louis, W.; Wu, C.; Cuff, P.; Wagner, S.; Sturm, J.C. An EEG Acquisition and Biomarker-Extraction System Using Low-Noise-Amplifier and Compressive-Sensing Circuits Based on Flexible, Thin-Film Electronics. IEEE J. Solid-State Circuits 2017, 52, 309. [CrossRef]

31. Abdulghani, A.; Abdulghani, A.; Casson, A.; Villegas, E. Compressive sensing scalp EEG signals: Implementations and practical performance. Med. Biol. Eng. Comput. 2012, 50, 1137-1145. [CrossRef] [PubMed]

32. Abualsaud, K.; Abualsaud, K.; Mahmuddin, M.; Hussein, R.; Mohamed, A. Performance evaluation for compression-accuracy trade-off using compressive sensing for EEG-based epileptic seizure detection in wireless tele-monitoring. In Proceedings of the 9th International Wireless Communications and Mobile Computing Conference (IWCMC), Sardinia, Italy, 1-5 July 2013.

33. Aghazadeh, R.; Aghazadeh, R.; Montagna, F.; Benatti, S.; Rossi, D.; Frounchi, J. Compressed Sensing Based Seizure Detection for an Ultra Low Power Multi-core Architecture. In Proceedings of the International Conference on High Performance Computing \& Simulation (HPCS), Orleans, France, 16-20 July 2018.

34. Shoaib, M.; Lee, K.; Jha, N.K.; Verma, N. A 0.6-107 / muW Energy-Scalable Processor for Directly Analyzing Compressively-Sensed EEG. IEEE Trans. Circuits Syst. Regul. Pap. 2014, 61, 1105-1118. [CrossRef]

35. Shoaran, M.; Schmid, A. A Power-Efficient Compressive Sensing Platform for Cortical Implants. In Efficient Sensor Interfaces, Advanced Amplifiers and Low Power RF Systems; Springer: Cham, Switzerland, 2016; pp. 103-122.

36. Mammone, N.; Salvo, S.; Bonanno, L.; Ieracitano, C.; Marino, S.; Marra, A.; Bramanti, A.; Morabito, F. Brain Network Analysis of Compressive Sensed High-Density EEG Signals in AD and MCI Subjects. IEEE Trans. Ind. Inf. 2019, 15, 527-536. [CrossRef]

37. Morabito, F.; Labate, D.; Bramanti, A.; Foresta, F.; Morabito, G.; Palamara, I.; Szu, H. Enhanced Compressibility of EEG Signal in Alzheimer's Disease Patients. IEEE Sens. J. 2013, 13, 3255-3262. [CrossRef]

38. Kaliannan, B.; Pasupureddi, V. A low power charge mode compressive acquisition of multichannel EEG signals. In Proceedings of the IEEE International Symposium on Circuits and Systems (ISCAS), Montreal, QC, Canada, 22-25 May 2016; pp. 518-521.

39. Hosseini, M.; Kamal, M.; Shoaran, M.; Leblebici, Y.; Schmid, A.; Vandergheynst, P. Compressive multichannel cortical signal recording. In Proceedings of the 2013 IEEE International Conference on Acoustics, Speech and Signal Processing, Vancouver, BC, Canada, 26-31 May 2013; pp. 4305-4309.

40. Shrivastwa, R.; Pudi, V.; Duo, C.; So, R.; Chattopadhyay, A.; Cuntai, G. A Brain-Computer Interface Framework Based on Compressive Sensing and Deep Learning. IEEE Consum. Electron. Mag. 2020, 9, 90-96. [CrossRef]

41. Lee, H.; Choi, J.; Kim, S.; Jun, S.; Lee, B. A Compressive Sensing-Based Automatic Sleep-Stage Classification System With Radial Basis Function Neural Network. IEEE Access 2019, 7, 186499-186509. [CrossRef]

42. Wen, D.; Jia, P.; Lian, Q.; Zhou, Y.; Lu, C. Review of Sparse Representation-Based Classification Methods on EEG Signal Processing for Epilepsy Detection, Brain-Computer Interface and Cognitive Impairment. Front. Aging Neurosci. 2016, 8, 172. [CrossRef]

43. Mohsina, M.; Majumdar, A. Gabor based analysis prior formulation for EEG signal reconstruction. Biomed. Signal Process. Control 2013, 8, 951-955. [CrossRef]

44. Aviyente, S. Compressed Sensing Framework for EEG Compression. In Proceedings of the IEEE SSP, Madison, WI, USA, 26-29 August 2007; pp. 181-184. 
45. Dao, P.; Griffin, X.; Li, X. Compressed Sensing of EEG with Gabor Dictionary: Effect of Time and Frequency Resolution. In Proceedings of the 40th Annual International Conference of the IEEE Engineering in Medicine and Biology Society (EMBC), Honolulu, HI, USA, 18-21 July 2018; pp. 3108-3111.

46. Dao, P.; Li, X.; Griffin, X. Quantitative Comparison of EEG Compressed Sensing using Gabor and K-SVD Dictionaries. In Proceedings of the 2018 IEEE 23rd International Conference on Digital Signal Processing (DSP), Shanghai, China, 19-21 November 2018.

47. Kuś, R.; Różański, P.T.; Durka, P.J. Multivariate matching pursuit in optimal Gabor dictionaries: Theory and software with interface for EEG/MEG via Svarog. Biomed. Eng. Online 2013, 12, 94. [CrossRef] [PubMed]

48. Zhang, H.; Chen, C.; Wu, Y.; Li, P. Decomposition and compression for ECG and EEG signals with sequence index coding method based on matching pursuit. China Univ. Posts Telecommun. 2012, 19, 92-95. [CrossRef]

49. Chen, L.; Zhao, E.; Wang, D.; Han, Z.; Zhang, S.; Xu, C. Feature extraction of EEG signals from epilepsy patients based on Gabor transform and EMD decomposition. In Proceedings of the 2010 Sixth International Conference on Natural Computation, Yantai, China, 10-12 August 2010; pp. 1243-1247.

50. Quian, R.; Blanco, S.; Rosso, O.; Garcia, H.; Rabinowicz, A. Searching for hidden information with Gabor transform in generalized tonic-clonic seizures Electroencephalography. Clin. Neurophysiol. 1997, 103, 434-439. [CrossRef]

51. Zhang, Z.; Jung, T.; Makeig, S.; Rao, B. Compressed Sensing of EEG for Wireless Telemonitoring With Low Energy Consumption and Inexpensive Hardware. IEEE Trans. Biomed. Eng. 2013, 60, 221-224. [CrossRef] [PubMed]

52. Birvinskas D.; Vacius, J.; Ignas, M.; Robertas, D. Fast DCT Algorithms for EEG Data Compression in Embedded Systems. Comput. Sci. Inf. Syst. 2014, 12, 49-62. [CrossRef]

53. Latka, M.; Was, Z.; Kozik, A.; West, B. Wavelet analysis of epileptic spikes. Phys. Rev. E 2003, 67, 052902. [CrossRef] [PubMed]

54. Fira, M.; Maiorescu, V.; Goras, L. The Analysis of the Specific Dictionaries for Compressive Sensing of EEG Signals. In Proceedings of the Ninth International Conference on Advances in Computer-Human Interactions, Venice, Italy, 24-28 April 2016.

55. Andrle, M.; Neira, A. Cardinal B-spline dictionaries on a compact interva. Appl. Comput. Harmon. Anal. 2005, 18, 336-346. [CrossRef]

56. Andrle, M.; Neira, A. From cardinal spline wavelet bases to highly coherent dictionaries. J. Phys. A Math. Theory 2008, 41, 172001. [CrossRef]

57. Andrle, M.; Neira, A. Spline wavelet dictionaries for non-linear signal approximation. In Proceedings of the International Conference on Interactions between Wavelets and Splines, Athens, Greece, 16-19 May 2005.

58. Craven, D.; McGinley, B.; Kilmartin, L.; Glavin, M.; Jones, E. Compressed Sensing for Bioelectric Signals: A Review. IEEE J. Biomed. Health Inf. 2015, 19, 529-540. [CrossRef] [PubMed]

59. Mahrous, H.; Ward, R. A Low Power Dirac Basis Compressed Sensing Framework for EEG using a Meyer Wavelet Function Dictionary. In Proceedings of the 2016 IEEE Canadian Conference on Electrical and Computer Engineering (CCECE), Vancouver, BC, Canada, 15-18 May 2016.

60. Zhu, J.; Chen, C.; Su, S.; Chang, Z. Compressive Sensing of Multichannel EEG Signals via $l_{q}$ Norm and Schatten- p Norm Regularization. Math. Probl. Eng. 2016, 2016, 208-216. [CrossRef]

61. Qiu, S.; Li, Z.; He, W.; Zhang, L.; Yang, C.; Su, C. Brain-Machine Interface and Visual Compressive Sensing-Based Teleoperation Control of an Exoskeleton Robot. IEEE Trans. Fuzzy Syst. 2017, 25, 58-69. [CrossRef]

62. Liu, H.; Zhang, T.; Ye, Y.; Pan, C.; Yang, G.; Wang, J.; Qiu, R. A Data Driven Approach for Resting-state EEG signal Classificition of Schizophrenia with Control Participants Using Random Matrix Theory. arXiv 2017, arXiv:1712.05289.

63. Fauvel, S.; Ward, R. An energy efficient compressed sensing framework for the compression of electroencephalogram signals. Sensors 2014, 14, 1474-1496. [CrossRef] [PubMed]

64. Sheng, J.; Yang, C.; Herbordt, M. Hardware-efficient compressed sensing encoder designs for WBSNs. In Proceedings of the IEEE High Performance Extreme Computing Conference (HPEC), Waltham, MA, USA, 15-17 September 2015.

65. Tello, R.; Pant, J.; Müller, S.M.; Krishnan, S.; Bastos-Filho, T.F. An Evaluation of Performance for an Independent SSVEP-BCI Based on Compressive Sensing System; In Proceedings of the World Congress on Medical Physics and Biomedical Engineering, Toronto, ON, Canada, 7-12 June 2015. 
66. Gilbert, A.; Indyk, P. Sparse recovery using sparse matrices. IEEE Proc. 2010, 98, 937-947. [CrossRef]

67. Li, X.; Dao, P.; Griffin, A. Effect of Epoch Length on Compressed Sensing of EEG. In Proceedings of the 40th Annual International Conference of the IEEE Engineering in Medicine and Biology Society (EMBC), Honolulu, HI, USA, 18-21 July 2018; pp. 1-4.

68. Mahrous, H.; Ward, R. Block Sparse Compressed Sensing of Electroencephalogram (EEG) Signals by Exploiting Linear and Non-Linear Dependencies. Sensors 2016, 16, 201. [CrossRef] [PubMed]

69. Hanafy, M.; Ali, H.; Shaalan, H. Rakeness with block sparse Bayesian learning for efficient ZigBee-based EEG telemonitoring. Int. J. Commun. Syst. 2020, 33, 4219. [CrossRef]

70. Majumdar, A.; Shukla, A.; Ward, R. Combining Sparsity with Rank-Deficiency for Energy Efficient EEG Sensing and Transmission over Wireless Body Area Network. In Proceedings of the IEEE International Conference on Acoustics, Speech and Signal Processing (ICASSP), Brisbane, QLD, Australia, 19-24 April 2015; pp. 837-841.

71. Nguyen, T.; Shin, Y. Deterministic Sensing Matrices in Compressive Sensing: A Survey. Sci. World J. 2013, 2013, 192795. [CrossRef] [PubMed]

72. Monajemi, H.; Jampana, P.; Sastry, C. Deterministic compressed sensing matrices. Proc. Natl. Acad. Sci. USA 2013, 110, 1181-1186. [CrossRef] [PubMed]

73. Liu, X.; Xia, S. Constructions of quasi-cyclic measurement matrices based on array codes. In Proceedings of the 2013 IEEE International Symposium on Information Theory, Istanbul, Turkey, 7-12 July 2013.

74. Liu, X.; Xia, S.; Dai, T. Deterministic constructions of binary measurement matrices with various sizes. In Proceedings of the 2015 IEEE International Conference on Acoustics, Speech and Signal Processing (ICASSP), Brisbane, Australia, 19-24 April 2015.

75. Zhao, W.; Sun, B.; Wu, T.; Yang, Z. On-Chip Neural Data Compression Based On Compressed Sensing With Sparse Sensing Matrices. IEEE Trans. Biomed. Circuits Syst. 2018, 12, 242-254. [CrossRef] [PubMed]

76. Zhao, W.; Sun, B.; Wu, T.; Yang, Z. Hardware efficient, deterministic QCAC matrix based compressed sensing encoder architecture for wireless neural recording application. In Proceedings of the 2016 IEEE Biomedical Circuits and Systems Conference (BioCAS), Shanghai, China, 17-19 October 2016.

77. Fira, M.; Goras, L. Comparison of Inter-and Intra-Subject Variability of P300 Spelling Dictionary in EEG Compressed Sensing. Int. J. Adv. Comput. Sci. Appl. 2016, 7, 366-371. [CrossRef]

78. Gramfort, A.; Kowalski, M.; Hämäläinen, M. Mixed-norm estimates for the M/EEG inverse problem using accelerated gradient methods. Phys. Med. Biol. 2012, 57, 1937-1961. [CrossRef] [PubMed]

79. Liu, Y.; Vos, M.; Huffel, S. Compressed Sensing of Multichannel EEG Signals: The Simultaneous Cosparsity and Low-Rank Optimization. IEEE Trans. Biomed. Eng. 2015, 62, 2055-2061. [CrossRef] [PubMed]

80. Tayyib, M.; Amir, M.; Javed, U.; Akram, M.W.; Yousufi, M.; Qureshi, I.M.; Ullah, H. Accelerated sparsity based reconstruction of compressively sensed multichannel EEG signals. PLOS ONE 2020, 15, e0225397. [CrossRef] [PubMed]

81. Zou, X.; Feng, L.; Sun, H. Compressive Sensing of Multichannel EEG Signals Based on Graph Fourier Transform and Cosparsity. Neural Process. Lett. 2020, 51, 1227-1236. [CrossRef]

82. Zou, X.; Feng, L.; Sun, H. Robust compressive sensing of multichannel EEG signals in the presence of impulsive noise. Inf. Sci. 2018, 429, 120-129. [CrossRef]

83. Zhang, Z.; Jung, T.; Makeig, S.; Pi, Z.; Rao, B. Spatiotemporal Sparse Bayesian Learning With Applications to Compressed Sensing of Multichannel Physiological Signals. IEEE Trans. Neural Syst. Rehabil. Eng. 2014, 22, 1186-1197. [CrossRef] [PubMed]

84. Liua, B.; Zhang, Z.; Xu, G.; Feng, H.; Fu, Q. Energy efficient telemonitoring of physiological signals via compressed sensing: A fast algorithm and power consumption evaluation. Biomed. Signal Process. Control 2014, 11, 80-88. [CrossRef]

85. Duarte, M.F.; Sarvotham, S.; Baron, D.; Wakin, M.B.; Baraniuk, R.G. Distributed Compressed Sensing of Jointly Sparse Signals. In Proceedings of the Conference Record of the Thirty-Ninth Asilomar Conference on Signals, Systems and Computers, Pacific Grove, CA, USA, 30 October-2 November 2005; pp. 1537-1541.

86. Djelouat, H.; Baali, H.; Amira, A.; Bensaali, F. Joint sparsity recovery for compressive sensing based EEG system. In Proceedings of the IEEE 17th International Conference on Ubiquitous Wireless Broadband (ICUWB), Salamanca, Spain, 12-15 September 2017. 
87. Khoshnevis, S.; Ghorshi, S. Recovery of Event Related Potential Signals using Compressive Sensing and Kronecker Technique. In Proceedings of the IEEE Global Conference on Signal and Information Processing (GlobalSIP), Ottawa, ON, Canada, 11-14 November 2019.

88. Zhang, Z. Comparison of Sparse Signal Recovery Algorithms with Highly Coherent Dictionary Matrices: The Advantage of T-MSBL. Res. Note 2012.

89. Rani, M.; Dhok, S.; Deshmukh, R. EEG Monitoring: Performance Comparison of Compressive Sensing Reconstruction Algorithms. In Advances in Intelligent Systems and Computing; Springer: Singapore, 2019; p. 862.

90. Gurve, D.; Pant, J.; Krishnan, S. Real-time fetal ECG extraction from multichannel abdominal ECG using compressive sensing and ICA. In Proceedings of the 39th Annual International Conference of the IEEE Engineering in Medicine and Biology Society (EMBC), Seogwipo, Korea, 11-15 July 2017.

91. Arjoune, Y.; Kaabouch, N.; El Ghazi, H.; Tamtaoui, A. Compressive sensing: Performance comparison of sparse recovery algorithms. In Proceedings of the IEEE 7th Annual Computing and Communication Workshop and Conference (CCWC), Las Vegas, NV, USA, 9-11 January 2017.

92. Chen, Y.; Lin, H.; Chiu, H.; Ma, H. A compressive sensing framework for electromyogram and electroencephalogram. In Proceedings of the IEEE International Symposium on Medical Measurements and Applications (MeMeA), Lisboa, Portugal, 11-12 June 2014.

93. Pilastri, A.; Manuel, J.; Tavares, R. Reconstruction Algorithms in Compressive Sensing : An Overview. In Proceedings of the Doctoral Symposium in Informatics and Telecommunications Engineering, Porto, Portugal, 3 February 2016.

94. Braun, H.; Turaga, P.; Spanias, A.; Katoch, S.; Jayasuriya, S.; Tepedelenlioglu, C. Reconstruction-Free Compressive Vision for Surveillance Applications. Synth. Lect. Signal Process. 2019, 10, 1-100. [CrossRef]

95. Kulkarni, K.; Turaga, P. Reconstruction-free action inference from compressive imagers. IEEE Trans. Pattern Anal. Mach. Intell. 2015, 38, 772-784 [CrossRef] [PubMed]

96. Paskov, S.; West, R.; Mitchell, J.; Hastie, T. Compressive Feature Learning. Neural Inf. Process. Syst. 2013, 26, 2931-2939.

97. Lohit, S.; Kulkarni, K.; Turaga, P.; Wang, J.; Sankaranarayanan, A. Reconstruction-free inference on compressive measurements. In Proceedings of the International Conference on Computational Cameras and Displays, Boston, MA, USA, 7-12 June 2015.

98. Lohit, S.; Kulkarni, K.; Turaga, P. Direct inference on compressive measurements using convolutional neural networks. In Proceedings of the IEEE International Conference on Image Processing (ICIP), Phoenix, AZ, USA, 25-28 September 2016; pp. 1913-1917.

99. Braun, H.; Turaga, P.; Spanias, A.; Tepedelenlioglu, C. Direct classification from compressively sensed images via deep Boltzmann machine. In Proceedings of the IEEE Asilomar Conference on Signals, Systems and Computers, Pacific Grove, CA, USA, 6-9 November 2016; pp. 454-457.

100. Fira, M. The EEG Signal Classification In Compressed Sensing Space. In Proceedings of the Twelfth International Multi-Conference on Computing in the Global Information Technology, Nice, France, 23-27 July 2017.

101. Kasabov, N.; Dhoble, K.; Nuntalid, N.; Indiveri, G. Dynamic evolving spiking neural networks for on-line spatio- and spectro-temporal pattern recognition. Neural Netw. 2013, 41, 188-201. [CrossRef] [PubMed]

102. Vato, A.; Bonzano, L.; Chiappalone, M.; Novellino, A.; Cicero, S.; Morabito, F.; Stillo, G. Spike manager: A new tool for spontaneous and evoked neuronal networks activity characterization. Neurocomputing 2004, 58-60, 1153-1161. [CrossRef]

103. Rodriguez, D.; Cardoso, V.; Gurve, D.; Loterio, F.; Laiseca, M.; Krishnan, S.; Filho, T. System based on subject-specific bands to recognize pedaling motor imagery: Towards a BCI for lower-limb rehabilitation. J. Neural Eng. 2019, 5, 6005.

104. Gurve, D.; Rodriguez, D.; Laiseca, M.; Cardoso, V.; Loterio, F.; Bastos, T.; Krishnan, S. Subject-specific EEG channel selection using non-negative matrix factorization for lower-limb motor imagery recognition. $J$. Neural Eng. 2020, 2, 6029. [CrossRef] [PubMed]

105. Gurve, D.; Rodriguez, D.; Bastos, T.; Krishnan, S. Motor Imagery Classification with Covariance Matrices and Non-Negative Matrix Factorization. In Proceedings of the 41st Annual International Conference of the IEEE Engineering in Medicine and Biology Society (EMBC), Berlin, Germany, 23-27 July 2019. 
106. Vourvopoulos, A.; Liarokapis, F. Navigation using braincomputer interfaces. In Proceedings of the IEEE 11th International Conference onTrust, Security and Privacy in Computing and Communications, Liverpool, UK, 25-27 June 2012.

107. Van, B.; Gürkök, H.; Plass-Oude, D.; Poel, M.; Nijholt, A. Experiencing BCI control in a popular computer game. IEEE Trans. Comput. Int. AI Games 2013, 5, 176-184.

108. Lin, J.; Tsai, S.; Ko, L. EEG-based learning system for online motion sickness level estimation in a dynamic vehicle environment. IEEE Trans. Neural Netw. Learn. Syst. 2013, 24, 1689-1700.

109. Ko, L.; Lee, H.; Tsai, S.; Shih, T.; Chuang, Y.; Huang, H.; Ho, S.; Lin, C. EEG-based motion sickness classification system with genetic feature selection.In Proceedings of the IEEE Symposium on Computational Intelligence, Cognitive Algorithms, Mind, and Brain (CCMB), Singapore, 16-19 April 2013.

110. Fukushima, M.; Inoue, A.; Niwa, T. Emotional evaluation of $\mathrm{tv}-\mathrm{cm}$ using the fractal dimension and the largest lyapunov exponent. In Proceedings of the IEEE International Conference on Systems Man and Cybernetics (SMC), Istanbul, Turkey, 10-13 October 2010.

111. Venthur, B.; Blankertz, B.; Gugler, M.F.; Curio, G. Novel applications of bci technology: Psychophysiological optimization of working conditions in industry. In Proceedings of the IEEE International Conference on Systems Man and Cybernetics (SMC), Istanbul, Turkey, 10-13 October 2010.

112. Su, F.; Zhou, H.; Feng, Z.; Ma, J. A biometric-based covert warning system using EEG. In Proceedings of the International Conference on Biometrics (ICB), New Delhi, India, 29 March-1 April 2012.

113. Nakanishi, I.; Baba, S.; Li, S. Evaluation of brain waves as biometrics for driver authentication using simplified driving simulator. In Proceedings of the International Conference on Biometrics and Kansei Engineering, Takamatsu, Kagawa, Japan, 19-22 September 2011.

114. Tan, D.S.; Nijholt, A. Brain-computer interfaces: Applying our minds to human-computer interaction. In Enhancing Human-Computer Interaction with Input from Active and Passive Brain-Computer Interfaces; Springer: London, UK, 2010; pp. 181-199.

115. Bonnet, L.; Lotte, F.; Lécuyer, A. Two brains one game: Design and evaluation of a multi-user bci video game based on motor imagery. IEEE Trans. Comput. Int. AI Games 2013, 5, 185-198. [CrossRef]

(C) 2020 by the authors. Licensee MDPI, Basel, Switzerland. This article is an open access article distributed under the terms and conditions of the Creative Commons Attribution (CC BY) license (http://creativecommons.org/licenses/by/4.0/). 